\title{
Synthesis of Chalcone Derivatives
}

\section{Xiaobo Liu ${ }^{1}$, Xiaojie Liu ${ }^{1}$ and Yinhua Xiong ${ }^{1, a^{*}}$}

${ }^{1}$ School of Pharmacy, Jiangxi Science \& Technology Normal University, Nanchang 330013, China

axiongyhfriend@126.com

*The corresponding author

\section{Keywords: Synthesis; Chalcone; Acetophenone}

\begin{abstract}
Chalcone is an important intermediate in many biologically active compounds such as flavonoid. Chalcone was prepared by the aldol condensation of benzaldehyde with acetophenone. In this paper, five novel chalcone derivatives were prepared. The structure was confirmed by MS and ${ }^{1}$ H NMR. Furthermore, the synthetic method was optimized. The yield of the step was at least $85 \%$ or more.
\end{abstract}

\section{Introduction}

Flavonoids have a wide range of applications, first of all it is a strong antioxidant, which can effectively remove oxygen free radicals in the body[1]. Second, Favonoids can not only improve blood circulation to achieve the purpose of lowering cholesterol, but also can effectively reduce the incidence of cardiovascular and cerebrovascular diseases and alleviate their symptoms. More, flavonoids also inhibit inflammation to relieve pain, which is useful for the treatment of various types of allergic symptoms [2-4]. Its also has important pharmacological effects, such as Laliberte R reported anti-insect effect of Chalcone derivatives, He Keqin, Cheng Guifang and other reports Chalcone derivatives have anti-allergic effects and anti-cancer effect. Many quinazoline derivatives which exhibited potential biological activities, such as 3-oxo-1,3-diphenylpropyl-pyrrolidine-1-carbodithioate (7-methyl-2-pheny-lH-imidazo[1,2-a]pyridin-3-yl)(phenyl)methanone (2) [5], 4-acetyl-1,3-diphenylhexane-1,5-dione 3-(1-methyl-1H-indol-3-yl)-1,3-diphenylpropan-1-one 2-(diphenylmethyleneamino)-5-oxo-3,5-diphenylpentanoate(6)[9], biological activity.

Most of the synthetic methods of chalcone which reported in the literature have the drawbacks such as longer synthetic route, lower yield and harmful to environment. Therefore, the optimization of the synthetic route and methods of chalcone is necessary. In this study, we prepared five kinds of chalcone derivatives, and design and optimize the method of the sythesis of chalcone, making it more suitable for industrial production. The structures of representative chalcone derivatives were shown in Fig. 1.

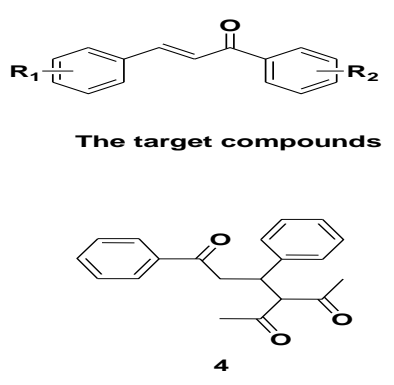

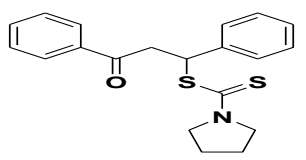

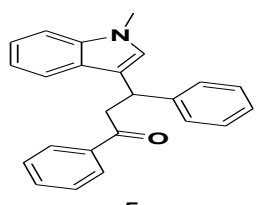

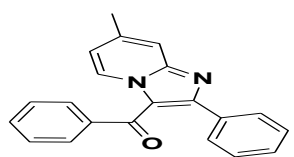

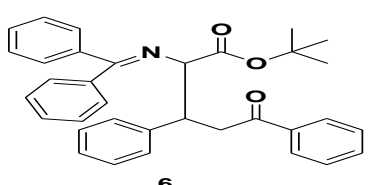

Figure 1. Structure of representative chalcone derivatives 


\section{Materials and Methods}

NMR spectra were performed using Bruker $400 \mathrm{MHz}$ spectrometers (Bruker Bioscience, Billerica, MA, USA) with TMS as an internal standard. Mass spectra (MS) were taken in ESI mode on Agilent 1100 LC-MS (Agilent, Palo Alto, CA, USA). All the materials were obtained from commercial suppliers and used without purification, unless otherwise specified. Yields were not optimized. TLC analysis was carried out on silica gel plates GF254 (Qindao Haiyang Chemical, China).

\section{Synthesis of Compounds}

The structures and the synthetic route were shown in Scheme 1.
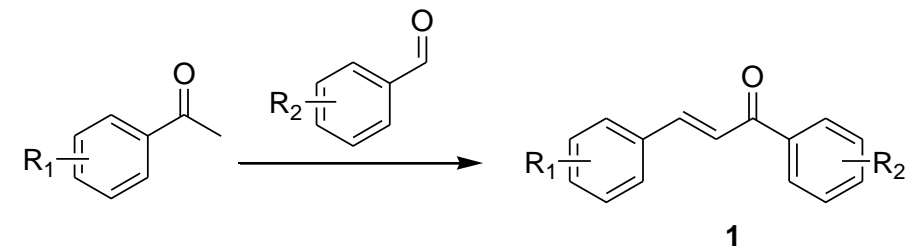

1a: $\mathrm{R}_{1}=\mathrm{H} ; \mathrm{R}_{2}=\mathrm{H}$
1b: $\mathrm{R}_{1}=2-\mathrm{Cl}-3-\mathrm{Cl} ; \mathrm{R}_{2}=4-\mathrm{CH}_{3}$
1c: $\mathrm{R}_{1}=3-\mathrm{Br} ; \mathrm{R}_{2}=4-\mathrm{CH}_{3}$
1d: $\mathrm{R}_{1}=\mathrm{Br} ; \mathrm{R}_{2}=\mathrm{H}$
1e: $\mathrm{R}_{1}=2-\mathrm{F} ; \mathrm{R}_{2}=4-\mathrm{F}$

Scheme 1. The synthetic route of compound 1a-1e

Reagents and Conditions: ethanol, $10 \% \mathrm{NaOH}$.

Chalcone(1a). A solution of acetophenone $(1.21 \mathrm{~g}, 0.01 \mathrm{~mol})$ and benzaldehyde $(1.06 \mathrm{~g}, 0.01$ mol) in ethanol $(30 \mathrm{~mL})$. Then $10 \% \mathrm{NaOH}(1 \mathrm{~mL})$ was added slowly with stirring. After stirring at room temperature for $24 \mathrm{~h}$, the reaction was complete by TLC analysis. The mixture was then filtered, the filter cake was washed and recrystallized from ethanol. Residue was dried to obtain a powdery solid (1.91 g, 92\%). ESI -MS m/z: 209.1 [M+H] +. 1H NMR (400 MHz, DMSO) $\delta 8.14(\mathrm{~d}$, $\mathrm{J}=7.3 \mathrm{~Hz}, 2 \mathrm{H}), 7.93(\mathrm{t}, \mathrm{J}=10.3 \mathrm{~Hz}, 1 \mathrm{H}), 7.88(\mathrm{dd}, \mathrm{J}=6.6,2.8 \mathrm{~Hz}, 2 \mathrm{H}), 7.78-7.70(\mathrm{~m}, 1 \mathrm{H}), 7.66$ $(\mathrm{t}, \mathrm{J}=7.3 \mathrm{~Hz}, 1 \mathrm{H}), 7.56(\mathrm{t}, \mathrm{J}=7.6 \mathrm{~Hz}, 2 \mathrm{H}), 7.50-7.42(\mathrm{~m}, 3 \mathrm{H})$.

3-(2,3-dichlorophenyl)-1-p-tolylprop-2-en-1-one(1b). Experimental operation as (1a). (2.53 g, 87.5\%). ESI -MS m/z: $291.0[\mathrm{M}+\mathrm{H}]^{+}{ }^{+}{ }^{1} \mathrm{H}$ NMR (400 MHz, DMSO) $\delta 7.43(\mathrm{~d}, J=1.8 \mathrm{~Hz}, 1 \mathrm{H})$, $7.22(\mathrm{dd}, J=16.8,11.9 \mathrm{~Hz}, 3 \mathrm{H}), 7.01(\mathrm{dd}, J=8.4,1.9 \mathrm{~Hz}, 1 \mathrm{H}), 6.84(\mathrm{t}, J=11.5 \mathrm{~Hz}, 2 \mathrm{H}), 6.52(\mathrm{~d}, J$ $=8.0 \mathrm{~Hz}, 2 \mathrm{H}), 1.54(\mathrm{~s}, 3 \mathrm{H})$.

3-(3-bromophenyl)-1-p-tolylprop-2-en-1-one(1c). Experimental operation as (1a). (2.67 g, 89.2\%). ESI -MS m/z: $302.0[\mathrm{M}+\mathrm{H}]^{+} .{ }^{1} \mathrm{H}$ NMR (400 MHz, DMSO) $\delta 8.06(\mathrm{~d}, J=8.1 \mathrm{~Hz}, 2 \mathrm{H}), 7.97$ $(\mathrm{d}, J=15.6 \mathrm{~Hz}, 1 \mathrm{H}), 7.85(\mathrm{~d}, J=8.4 \mathrm{~Hz}, 2 \mathrm{H}), 7.66(\mathrm{t}, J=12.7 \mathrm{~Hz}, 3 \mathrm{H}), 7.35(\mathrm{t}, J=13.7 \mathrm{~Hz}, 2 \mathrm{H})$, $2.37(\mathrm{~d}, J=15.8 \mathrm{~Hz}, 3 \mathrm{H})$.

3-(3-bromophenyl)-1-phenylprop-2-en-1-one(1d). Experimental operation as (1a). (2.66 g, 93.1\%). ESI -MS m/z: $288.0[\mathrm{M}+\mathrm{H}]^{+} .{ }^{1} \mathrm{H}$ NMR (400 MHz, DMSO) $\delta 8.15(\mathrm{~d}, J=7.3 \mathrm{~Hz}, 2 \mathrm{H}), 7.98$ $(\mathrm{d}, J=15.7 \mathrm{~Hz}, 1 \mathrm{H}), 7.85(\mathrm{~d}, J=8.5 \mathrm{~Hz}, 2 \mathrm{H}), 7.76-7.62(\mathrm{~m}, 4 \mathrm{H}), 7.56(\mathrm{t}, J=7.6 \mathrm{~Hz}, 2 \mathrm{H})$.

3-(3-fluorophenyl)-1-(4-fluorophenyl)prop-2-en-1-one(1e). Experimental operation as (1a). $(2.23$ g, $91.5 \%)$. ESI -MS m/z: $245.1[\mathrm{M}+\mathrm{H}]^{+} .{ }^{1} \mathrm{H}$ NMR $(400 \mathrm{MHz}, \mathrm{DMSO}) \delta 8.24$ (dd, $J=8.8,5.6$ $\mathrm{Hz}, 2 \mathrm{H}), 8.01-7.87(\mathrm{~m}, 3 \mathrm{H}), 7.74(\mathrm{~d}, J=15.6 \mathrm{~Hz}, 1 \mathrm{H}), 7.39(\mathrm{t}, J=8.8 \mathrm{~Hz}, 2 \mathrm{H}), 7.30(\mathrm{t}, J=8.8 \mathrm{~Hz}$, $2 \mathrm{H})$.

\section{Conclusions}

In conclusion, 5 kinds of chalcone derivatives were prepared by substituted acetophenone and substituted aromatic aldehydes by hydroxyaldehyde condensation. The synthetic method of chalcone and the reactions conditions were optimized, the yield of the product was much more higher, and making it more suitable for industrial production. It structure was confirmed by MS and $1 \mathrm{H}$ NMR spectrum. 


\section{Acknowledgments}

We gratefully acknowledge the generous support provided by Project supported by the Natural Science Foundation of Jiangxi Province (81660692), Doctoral Scientific Research Foundation of Jiangxi Science \& Technology Normal University and Science and Technology Project Founded by the Education Department of Jiangxi Province (No. GJJ14598).

\section{References}

[1] Ridgway T, Tucker G, Wiseman H. Novel bioconversions for the production of designer antioxidant and colourant flavonoids using polyphenol oxidases. Biotechnology and Genetic Engineering Reviews, 1997, 14(1):165-190.

[2] Yao Q, Gong Z, Su J, et al. Method for increasing yield of total flavonoids in Ganoderma lucidum mycelium:, US9284585. 2016.

[3] Peterson J J, Dwyer J T, Jacques P F, et al. Do Flavonoids Reduce Cardiovascular Disease Incidence or Mortality in US and European Populations? Nutrition Reviews, 2012.

[4] Stapleton A E, Walbot V. Flavonoids can protect maize DNA from the induction of ultraviolet radiation damage. Plant Physiology, 1994, 105(3):881-889.

[5] Karmakar B, Banerji J. An expedient, fast and competent synthesis of organic dithiocarbamates over nanocrystalline $\mathrm{MgO}$ in water at room temperature. ChemInform, 2012, 43(11):6584-6586.

[6] Yu Y, Liu Y, Liu A, et al. Ligand-free Cu-catalyzed [3 + 2] cyclization for the synthesis of pyrrolo[1,2-a]quinolines with ambient air as a terminal oxidant. Organic \& Biomolecular Chemistry, 2016, 14(31).

[7] Baruah B, Boruah A, Prajapati D, et al. BiCl 3, or Cdl 2, catalyzed Michael addition of 1,3-dicarbonyl compounds under microwave irradiations. Tetrahedron Letters, 1997, 38(8):1449-1450.

[8] Liang D, Li X, Zhang W, et al. ChemInform Abstract: Br 2, as a Novel Lewis Acid Catalyst for Friedel-Crafts Alkylation of Indoles with $\alpha, \beta$-Unsaturated Ketones. ChemInform, 2016, 57(27):1027-1030.

[9] Hua M Q, Wang L, Cui H F, et al. A powerful synergistic effect for highly efficient diastereo-and enantioselective phase-transfer catalyzed conjugate additions. Chemical Communications, 2011, 47(5): 1631-1633. 